\title{
Editorial
}

Radiologe 2013 $53: 4$

DOI 10.1007/s00117-012-2432-y

Online publiziert: 6 . Januar 2013

(c) Springer-Verlag Berlin Heidelberg 2013

M.F. Reiser ${ }^{1} \cdot$ P. Mildenberger ${ }^{2}$

${ }^{1}$ Institut für Klinische Radiologie, Klinikum der Ludwig-Maximilians-Universität München, München

2 Klinik und Poliklinik für Diagnostische und Interventionelle Radiologie,

Universitätsmedizin der Johannes-Gutenberg-Universität Mainz, Mainz

\section{Informationstechnologie und Management in der Radiologie}

\section{Eine neue Rubrik in Der Radiologe}

Sehr geehrte Leserinnen und Leser,

wie kaum ein anderes Fach in der Medizin ist die Radiologie seit vielen Jahren durch die kontinuierlichen Entwicklungen der Informationstechnologie beeinflusst worden. Wesentliche Elemente waren hierbei die Einführung des Radiologieinformationssystems (RIS) und des Picture Archiving and Communication System (PACS) sowie die Computerunterstützung im Befundungsprozess. Die Abläufe in der Radiologie sind hier grundlegend verändert worden, Bilder und Befunde sind digital, oft schon nach kurzer Zeit, verfügbar. Für die Patienten hat dies meist deutliche Vorteile. Neben einer besseren Bildqualität ist durch die Optimierung der Kommunikationswege der Behandlungsablauf kürzer und v. a. sind Wiederholungen von Röntgenuntersuchungen damit weitgehend vermeidbar.

Die weitere Entwicklung im Bereich der Informationstechnologie wird auch in Zukunft die Radiologie nachhaltig prägen, u. a. werden die Teleradiologie und die weitergehende Vernetzung von Radiologien sowohl in Krankenhäusern als auch bei niedergelassenen Fachärzten spürbar zunehmen. Ebenso werden neue Verfahren der Bildbearbeitung unsere diagnostischen Verfahren beeinflussen, z. B. durch die automatisierte Erkennung von Organen oder pathologischen Bildmustern.
Parallel zu den Entwicklungen in der Informationstechnologie ist die Radiologie auch durch Entwicklungen und Änderungen im Gesundheitswesen allgemein gefordert. Dies bedingt eine intensivierte Beschäftigung mit Themen des Managements in der Radiologie. Unterschiedlichste Stichworte wie Qualität, Patientensicherheit, „benchmarking“, Workflowoptimierung oder Entscheidungsunterstützung für den Einsatz bildgebender Verfahren gehören dazu.

Diese neue Rubrik soll den Lesern von Der Radiologe aktuelle Entwicklungen und Hintergründe zu Themen aus den Bereichen Informationstechnologie und Management in kurzen und allgemein verständlichen Beiträgen vorstellen. Wir hoffen, dass dies auch einen Beitrag zur Verbesserung der Position der Radiologie in der Diskussion mit anderen Fachrichtungen und Gruppen leisten kann.

Im ersten Beitrag wird gezeigt, wie vielfältig bereits heute die radiologischen Informationen und Angebote im Internet sind.
Wir hoffen, dass die Beiträge dieser neuen Rubrik Ihr Interesse finden und freuen uns auf Rückmeldungen und Anregungen für weitere Themen.

Ihre

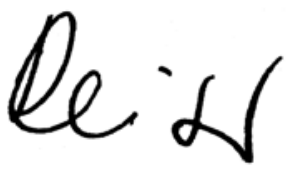

Prof. Dr. Dr. h.c. Maximilian F. Reiser

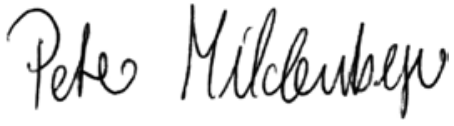

Prof. Dr. Peter Mildenberger

\section{Korrespondenzadresse}

Prof. Dr. P. Mildenberger

Klinik und Poliklinik für Diagnostische und Interventionelle Radiologie, Universitätsmedizin der Johannes-Gutenberg-Universität Mainz, Langenbeckstr. 1, 55131 Mainz peter.mildenberger@unimedizin-mainz.de 